\title{
Numerical and Experimental Validation of the Prototype of a Bio-Inspired Piping Inspection Robot
}

\author{
Swaminath Venkateswaran $1,2,+\left[\right.$, Damien Chablat ${ }^{1,3, *,+} \mathbb{C}$ and Frédéric Boyer ${ }^{1,4}$ \\ 1 Laboratoire des Sciences du Numérique de Nantes (LS2N), UMR CNRS 6004, 44300 Nantes, France; \\ swaminath.venkateswaran@1s2n.fr (S.V.); frederic.boyer@imt-atlantique.fr (F.B.) \\ 2 École Centrale de Nantes, 44321 Nantes, France \\ 3 Centre National de la Recherche Scientifique (CNRS), 44321 Nantes, France \\ 4 IMT Atlantique, 44307 Nantes, France \\ * Correspondence: damien.chablat@cnrs.fr \\ + These authors contributed equally to this work.
}

Received: 10 March 2019; Accepted: 19 April 2019; Published: 23 April 2019

\begin{abstract}
Piping inspection robots are of greater importance for industries such as nuclear, chemical and sewage. Mechanisms having closed loop or tree-like structures can be employed in such pipelines owing to their adaptable structures. A bio-inspired caterpillar type piping inspection robot was developed at Laboratoire des Sciences du Numérique de Nantes (LS2N), France. Using DC motors and leg mechanisms, the robot accomplishes the locomotion of a caterpillar in six-steps. With the help of Coulomb's law of dry friction, a static force model was written and the contact forces between legs of robot and pipeline walls were determined. The actuator forces of the DC motors were then estimated under static phases for horizontal and vertical orientations of the pipeline. Experiments were then conducted on the prototype where the peak results of static force analysis for a given pipe diameter were set as threshold limits to attain static phases inside a test pipeline. The real-time actuator forces were estimated in experiments for similar orientations of the pipeline of static force models and they were found to be higher when compared to the numerical model.
\end{abstract}

Keywords: piping inspection; bio-inspired robot; static force analysis; coulomb's law

\section{Introduction}

In industries such as nuclear, chemical and sewage, it is difficult for a human being to perform inspection or interventions. Manual intervention could not only lead to long-term radiation effects but also pose a serious threat to human life. Piping inspection robots play an essential role in such scenarios as they reduce human effort and impose lesser risks to life. Generally, the locomotion principles of these robots can employ mechanical systems or can be inspired from animals. Kassim et al. [1] proposed a distinction between these two categories where the mechanical systems can use wheels and pulleys [2], telescopic system [3], impact modules [4] or natural peristalsis [5] to accomplish the locomotion. On the other hand, the bio-inspired systems can mimic their locomotions from animals such as earthworms [6], snakes [7], millipedes [8], lizards [9] or an octopus [10]. Presently, several piping inspection robots have been developed. Nayak et al. [11] proposed a screw type robot that is capable of working inside 127-152 mm diameter pipelines. Zhang et al. [12] developed a flexible inchworm type robot that can adapt itself to varying diameters of pipes. Kwon et al. [13] developed an inspection robot that employs a caterpillar module with four bar linkages to work inside $100 \mathrm{~mm}$ diameter pipelines with bends and junctions. However, the design and development of inspection robots for pipelines less than $100 \mathrm{~mm}$ diameter is a big challenge. In addition, these piping inspection robots encounter some key problems, which include: (i) movement of the robot inside straight or curved sections 
of pipe while towing a payload; (ii) accurate positioning of the robot on the zone to be intervened; (iii) effective communication between onboard sensors and central station; and (iv) performing the desired mechanical activity such as drilling by withstanding the load of the mounted device. A novel bio-inspired piping inspection robot that mimics the locomotion of a caterpillar was proposed and developed by Henry et al. and Chablat et al. [14,15]. This robot is capable of addressing the issue of design of inspection robots for pipelines having diameters less than $100 \mathrm{~mm}$. Using electrical actuators with leg mechanisms and spindle drive units, this robot accomplishes the motion of a caterpillar and can work inside 40-94 $\mathrm{mm}$ diameter pipelines. They studied three architectures for optimization via slot-follower, four-bar and six-bar mechanisms in [14] for the choice of leg mechanism for the robot. By following a Heuristic optimization [16] technique in MATLAB, which aims at minimizing the space occupied by the mechanism and maximizing the force transmission factor, the slot-follower mechanism was chosen by the authors of [14]. An overview of the static force model for the robot is presented in [15]. Owing to the low velocity of the system, the dynamic forces on the robot under locomotion are proven to be significantly smaller in [17]. In this article, we focus on the detailed study of static force analysis and how its results are used as inputs for carrying out experiments on the prototype developed at LS2N, France. The static force analysis aimed at determining the clamping forces using Coulomb's law of dry friction for various diameters and orientations of pipeline. Experiments were carried out on the prototype where the peak results of the numerical force model were set as threshold limits to attain static phases. The real-time forces and torques induced on the actuators under locomotion were then interpreted using a Force-control algorithm inside a $74 \mathrm{~mm}$ diameter straight pipe.

The outline of the article is as follows. The locomotion principle of the robot explaining how the caterpillar motion is accomplished and the kinematic equations for leg mechanism are presented in Section 2. The static force model is presented in Section 3. The results of static force analysis are discussed in Section 3.1. The experimental setup of the prototype and their results are emphasized in Sections 4 and 4.1. The article then ends with conclusions.

\section{Locomotion Principle and Kinematic Equations of the Robot}

The locomotion principle of the robot is inspired from a caterpillar. The motion of a caterpillar is subdivided into three modules: a central blocking module and two elongation modules with one at the front and the other at the rear. A classical way to mimic this locomotion can be accomplished with the help of pneumatic bellows for blocking and electric motors for elongation [18,19]. However, while moving inside nuclear power plant pipelines there exists dust particles which limits the use of pneumatic systems. Hence, electrical actuators are used in the robot. A comparative study by Henry et al. [14] proposes a mechanism that is capable of simulating the locomotion of a caterpillar which is mimicked by using three electrical actuators. A cross-sectional view of the robot inside a pipeline of radius $r$ is represented in Figure 1.



Figure 1. Cross section view of the robot representing the actuators and leg mechanisms inside a pipeline of radius $r$. 
In Figure 1, $O$ represents the reference point for the leg mechanisms. Active spindle drives are used in the front and rear modules to attain a prismatic motion. The stroke lengths of the prismatic link $A C$ is represented by $\rho$. Three sets of legs, each on the front and rear modules are mounted at angles of $2 \pi / 3$ radians. The leg mechanisms ensure tight contact with the walls of the pipeline under static and dynamic phases of the robot and they can adapt their stretch based on the diameter changes of pipeline. The central module in Figure 1 also uses an active spindle drive to accomplish the elongation/retraction process and its stroke length is represented by $\rho_{c}$. The slot-follower leg mechanism is mounted at Point $A$ (passive rotary joint) of the actuator. The leg can slide along Point $B$ (passive prismatic joint) and $l_{0}$ represents the length of $A B$. Each leg will have its contact at Point $P$ on the pipeline and $l_{1}$ represents its length. Owing to the motor unit used for the spindle drive, the legs are mounted at an offset of distance $e$ from the central axis. $l_{2}$ represents the distance between Points $B$ and $C$. This robot accomplishes the locomotion of caterpillar in six-steps, as represented in Figure 2.
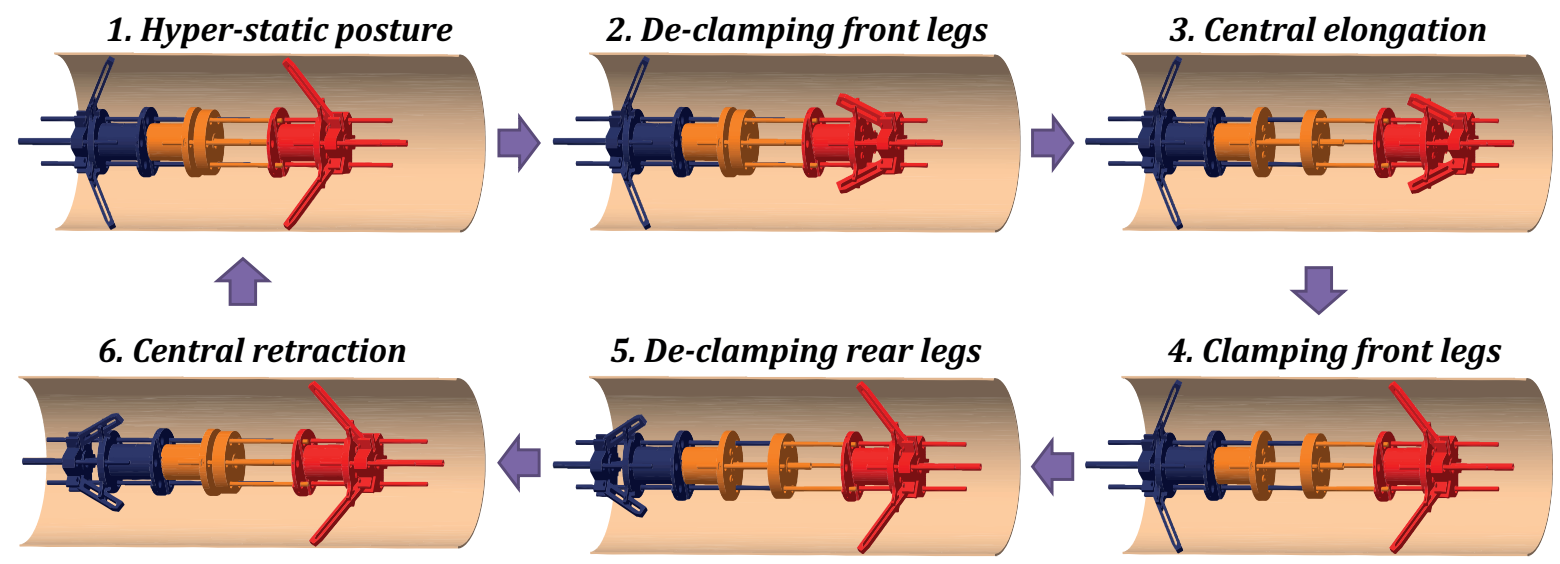

Figure 2. Demonstration of the six locomotion steps to attain caterpillar motion by the robot [20].

A hyper-static or over-constrained posture exists when both sets of legs of the robot are in contact with the walls. The coordinates $\left(z_{p}, y_{p}\right)$ of Point $P$ in Figure 1 can be written to obtain the closed-loop equation, which is given by:

$$
\begin{aligned}
& y_{p}=\frac{l_{2} l_{1}}{l_{0}}+e \\
& z_{p}=\frac{\rho l_{1}}{l_{0}}-\rho \quad \text { where } l_{0}=\sqrt{l_{2}^{2}+\rho^{2}}
\end{aligned}
$$

Equations (1) and (2) are used to derive the direct and inverse kinematic models (DKM and IKM) for the leg mechanism. For the DKM, for a known stroke length $\rho$ and dimensional parameters of leg, the radius of pipeline $r$ or $y_{p}$ can be estimated. For the inverse kinematic model, for a known diameter or radius of pipeline and dimensional parameters of leg, the actuation length $\rho$ can be estimated. The equations are derived using Maple and they are given by:

$$
\begin{aligned}
\text { DKM: } y_{P} & =\frac{l_{2}^{2} e+\rho^{2} e+l_{1} l_{2}}{\sqrt{l_{2}^{2}+\rho^{2}}} \\
\text { IKM: } \rho & =\frac{l_{2} \sqrt{l_{1}^{2}-e^{2}+2 e y_{P}-y_{P}^{2}}}{y_{P}-e}
\end{aligned}
$$

The Jacobian matrix can be estimated using these kinematic equations, with the help of which we can deduce the relation between contact forces and the actuator forces, which is given by: 


$$
F_{a}=\mathbf{J}^{T} F_{p}
$$

The estimation of actuator forces $F_{a}$ is presented in the upcoming sections.

\section{Static Force Analysis on the Robot}

For the calculation of forces on the motor during static phase, we consider two different models, which are depicted in Figure 3. In Model A, the rear leg modules are clamped with the pipeline walls and vice-versa in Model B.

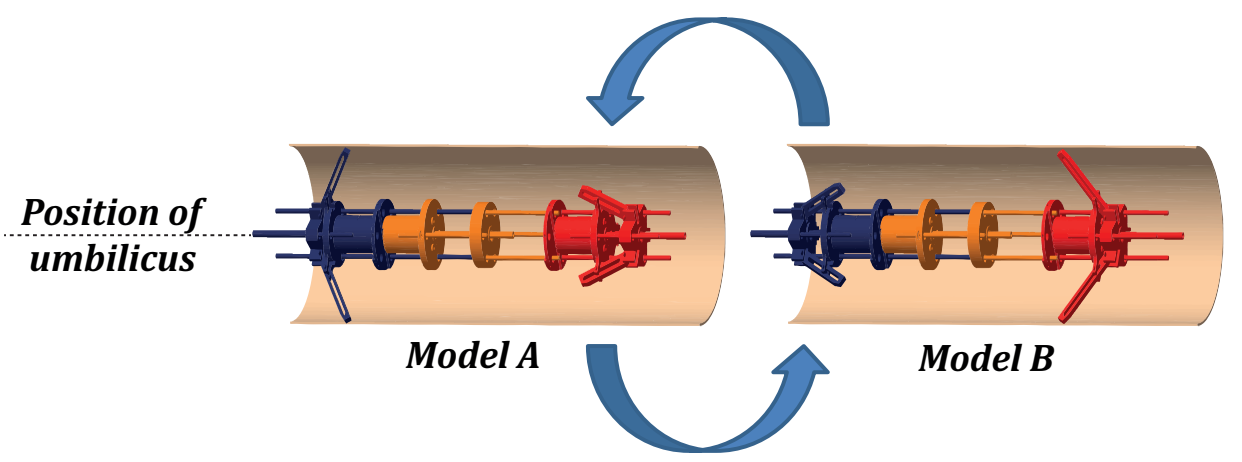

Figure 3. Two models of the robot considered for static force analysis of leg actuators.

The static algorithm mainly aims at estimating the contact forces between the robot and the pipeline. With the contact forces estimated, the forces induced on the actuators of the front (Model B) and rear modules (Model A) can be estimated. For the static force model, the robot is considered to be rigid and it can work only through straight pipelines having varying diameters. During the non-hyperstatic phases of clamping, the robot behaves similar to a cantilever beam where on one side three legs have three contact points with pipeline walls and on the free end the self-weight of robot generates a moment. The actuator forces and torques are affected by the orientations of the robot $(\theta)$ and the pipeline $(\alpha)$ (Figure 4$)$.

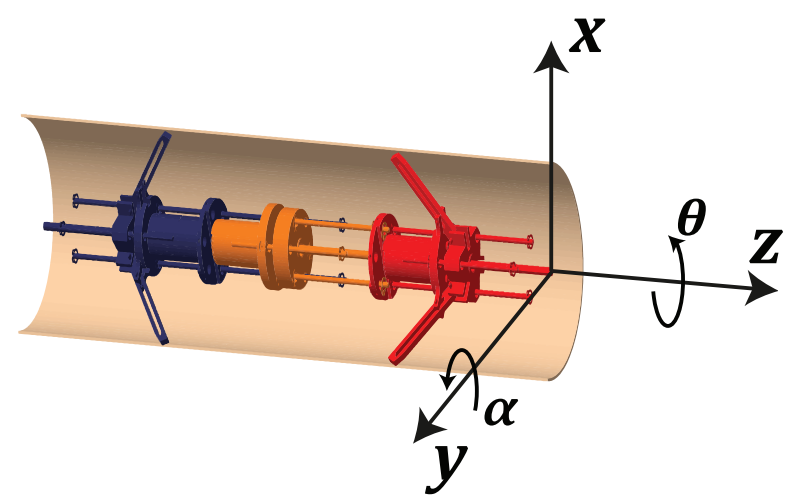

Figure 4. Representation of rotation angles of pipeline $(\alpha)$ and $\operatorname{robot}(\theta)$.

At every contact point, there exists three main forces between the legs and pipeline which are:

- $\quad N_{i}$-Normal Force

- $\quad T_{l i}$-Tangential longitudinal Force

- $\quad T_{r i}$-Tangential radial Force

The representation of these forces inside a pipeline of radius $r$ is shown in Figure 5 . 

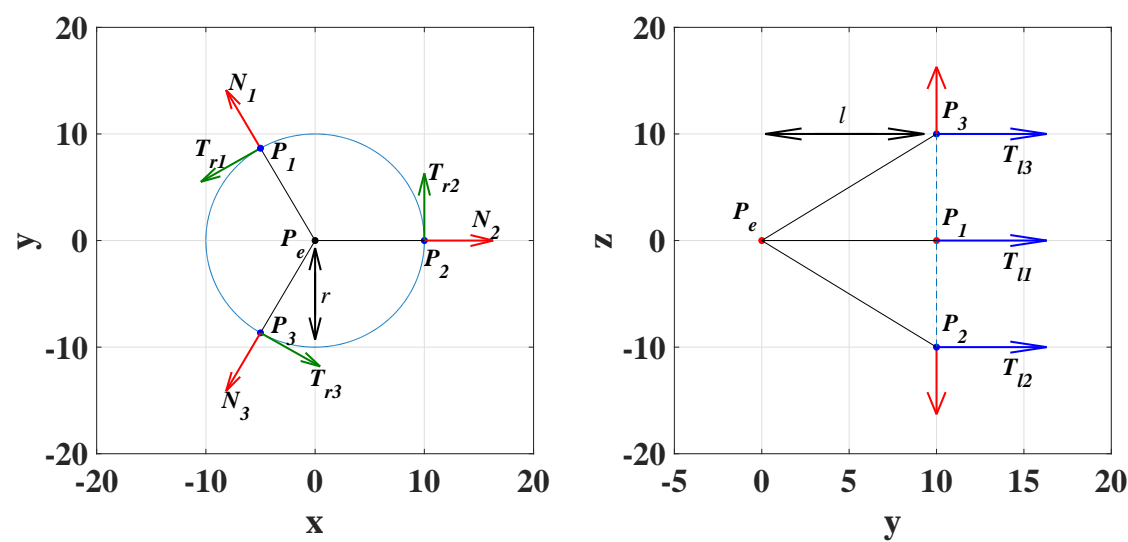

Figure 5. Schema of the contact forces during clamping as seen in $x-y$ (left) and $y-z$ (right) planes.

The static model equations from [15] have been revisited here. The contact circle, which consists of the force components, is depicted by the parameters $l$ and $r$. The rotation of robot about the $\mathrm{z}$-axis is represented by the parameter $\theta$ (not depicted in Figure 5). The position of center of gravity (CG) on z-axis is depicted by Point $P_{e}$ and the contact points are represented by $P_{1}, P_{2}$ and $P_{3}$. The vector coordinates are given by:

$$
\mathbf{p}_{e}=[0,0,0]^{T}
$$

where $\mathbf{p}_{e}$ represents the vector coordinates of $P_{e}$. The coordinates for the vector at the contact Points $P_{i}$ is given by:

$$
\mathbf{p}_{i}=\mathbf{R}_{z}(\theta) \mathbf{n}_{i}
$$

$$
\text { where } \mathbf{R}_{z}(\theta)=\left[\begin{array}{ccc}
\cos (\theta) & -\sin (\theta) & 0 \\
\sin (\theta) & \cos (\theta) & 0 \\
0 & 0 & 1
\end{array}\right] \quad \text { and } \mathbf{n}_{i}=\left[\begin{array}{c}
r \cos \left(\frac{2 \pi(i-1)}{3}\right) \\
r \sin \left(\frac{2 \pi(i-1)}{3}\right) \\
l
\end{array}\right] \quad \text { with } i=1,2,3
$$

In Equation (7), $\mathbf{n}_{i}$ are the coordinates of $P_{i}$ in the local reference frame of the contact points of legs. $\mathbf{R}_{z}$ is the rotation matrix of robot about z-axis. At the central Point $P_{e}$, there exists a wrench equation that is comprised of forces and moments and is given by the equation:

$$
\tau_{e}=\left[\begin{array}{c}
\mathbf{f}_{i} \\
\mathbf{m}_{i}
\end{array}\right]
$$

where $\mathbf{f}_{i}=\left[f_{i x}, f_{i y}, f_{i z}\right]^{T} \quad$ and $\mathbf{m}_{i}=\left[m_{i x}, m_{i y}, m_{i z}\right]^{T} \quad$ with $i=1,2,3$

According to the static equilibrium conditions, it is well known that:

$$
\sum_{i=1}^{3} f_{i}=0 \quad \text { and } \quad \sum_{i=1}^{3} m_{i}=0
$$

The tangential radial and longitudinal forces can be estimated directly from the reaction forces at three contact points of legs. The force vector at the contact points is given by: 


$$
\mathbf{f}_{i}=\left[\begin{array}{c}
T_{r i} \cos \left(\frac{2 \pi(i-1)}{3}+\frac{\pi}{2}\right) \\
T_{r i} \sin \left(\frac{2 \pi(i-1)}{3}+\frac{\pi}{2}\right) \\
T_{l i}
\end{array}\right] \quad \text { with } i=1,2,3
$$

At the contact Points $P_{i}$, there exists no moments and the wrench equation is comprised of only resultant forces and is represented by ${ }^{i} \tau_{i}$. About the central Point $P_{e}$, the wrench equation ${ }^{i} \tau_{0}$ can be calculated using the Varignon's theorem and is given by:

$$
{ }^{i} \tau_{0}={ }^{i} \tau_{i}+\left(\mathbf{p}_{i} \times \mathbf{f}_{i}\right) \quad \text { with } i=1,2,3
$$

By using Coulomb's law of dry friction, the normal forces can be estimated from the tangential radial and longitudinal forces. The equation is given by:

$$
N_{i} \geq \frac{\sqrt{T_{r i}^{2}+T_{l i}^{2}}}{\varphi} \quad \text { with } i=1,2,3
$$

Generally, the material used for nuclear or chemical pipeline is steel. For the legs of the robot, bronze is used as it has good mechanical properties such as self-lubrication and wear resistance. As the clamping forces could lead to deformations, a metallic alloy is considered over other materials such as ceramics or rubber. The coefficient of friction represented by $\varphi$ in Equation (12) is taken as 0.4 [15]. With the individual normal forces estimated, the global normal force $N_{\text {total }}$ can be estimated by summing the normal forces of each leg. $N_{\text {total }}$ also indicates the magnitude of contact forces $F_{p}$. The magnitude of actuator force $F_{a}$ can be estimated by using Equation (5), which is modified as:

$$
F_{a}=\eta_{f} N_{t o t a l}
$$

where $\eta_{f}$ is the force transmission factor that can be generated using the Jacobian matrix in Maple and is given by:

$$
\eta_{f}=\frac{(e-r)^{2} \sqrt{l_{1}^{2}-e^{2}+2 e r-r^{2}}}{l_{1}^{2} l_{2}}
$$

In Equation (11), the wrench model has dependencies on the position of CG and, in Equation (14), the factor $\eta_{f}$ has dependencies on the radius of the pipelines $r$. When the pipeline is horizontal or angled, the robot behaves similar to a cantilever beam and the distance between the clamped ends to the position of CG is essential to determine the forces induced on the actuators and this analysis is exploited in the next section.

\subsection{Results of Static Force Analysis}

In reality, inside an industrial pipeline, there exists many bends or curves. An example of various types of profiles that could be encountered by a piping inspection robot is shown in Figure 6 . 


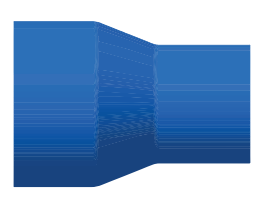

(a)

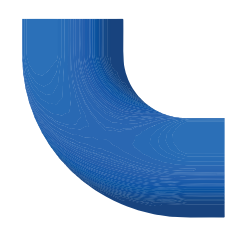

(b)

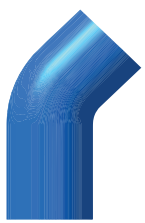

(c)

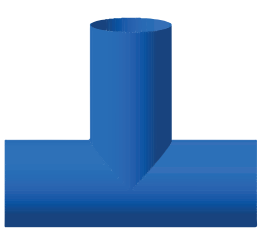

(d)

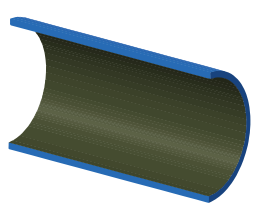

(e)

Figure 6. Possible profiles encountered by an inspection robot inside an industrial pipeline: (a) varying diameter; (b) curvature; (c) inclination; (d) branching; and (e) uneven surface walls [21].

The material used for elbows are usually stainless steel of grade Z401R and the connecting elements are stainless steel of grade Z2 CN18 10. These materials are non-magnetic and, due to continuous operation of facilities, there exists deposits on the inner walls of the pipeline (Figure 6e). In our case study, a straight pipeline of $2.5 \mathrm{~m}$ length with inner diameter range from 54 to $74 \mathrm{~mm}$ was considered. A Maxon motor GP 16 S ( $\phi 16)$ spindle drive unit coupled with an EC-max $(\phi 16)$ brushless motor [22] was used in each individual module of the robot for actuation. The spindle drive offers a maximum static axial load of $500 \mathrm{~N}$. The key specifications of the spindle drive and the DC-motor unit are listed in Appendix A Tables A1 and A2. The optimized dimensions of slot-follower leg mechanism is determined with respect to the spindle drive used for actuation in [14] by using Genetic Algorithm [23] in MATLAB. The objective functions aims at minimizing space occupied by the legs of the robot and maximizing the force transmission factor. The optimized dimensions for the legs used in the robot are provided in Table 1.

Table 1. Optimized dimensions of the slot-follower leg mechanism [15].

\begin{tabular}{cc}
\hline Lengths & Dimensions [mm] \\
\hline$l_{1}$ & 57 \\
$l_{2}$ & 7 \\
$e$ & 11 \\
$\rho$ & $8.5-45.5$ \\
\hline
\end{tabular}

Using the spindle motors, the leg mechanisms, coupling screws and standard fasteners such as circlips, studs, nuts and bolts, the entire robotic system was assembled. For the electronic unit, the ESCON 36/3 EC servo controller system offered by Maxon was used, which provides speed and torque control. The entire locomotion sequence is controlled by this control unit over the DC-Motors and the spindle drives through the umbilicus (robot cables). The digital model of the robot was realized using CATIA software and is represented in Figure 7. 


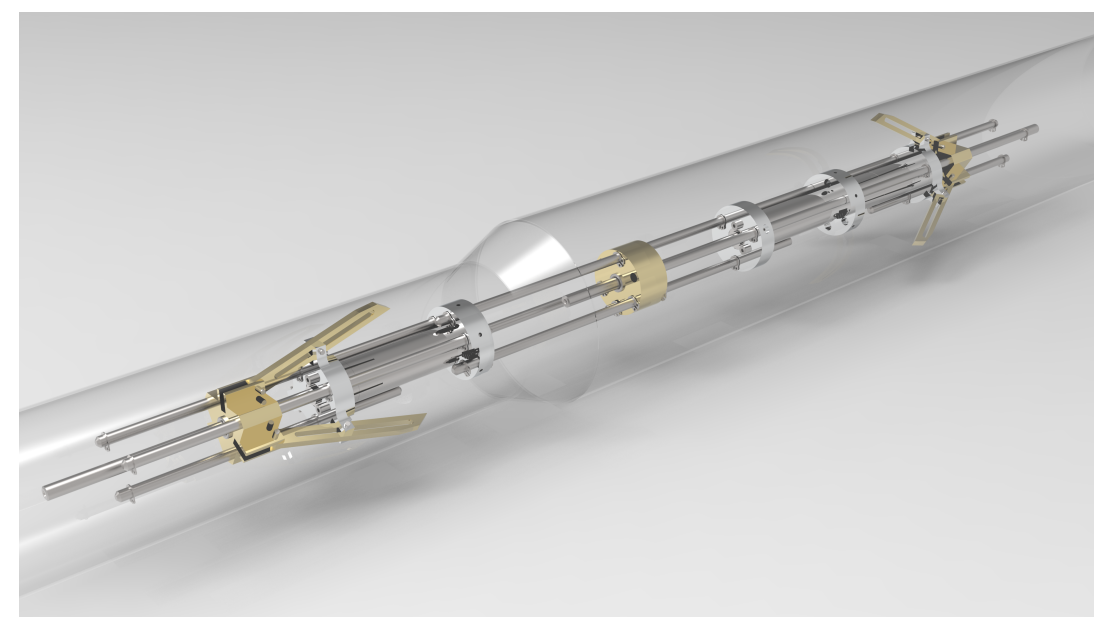

Figure 7. Digital model of the robot in CATIA software [17].

Forces Inside Horizontal and Vertical Orientations of Pipeline

The actuator forces of front and rear modules for horizontal and vertical orientations of pipeline were determined. With the help of Maple, the vector equations of the tangential radial and longitudinal forces were extracted, and are given by:

$$
\begin{gathered}
\mathbf{t}_{l i}=\left[T_{l 1}, T_{l 2}, T_{l 3}\right]^{T}=\left[\begin{array}{c}
\frac{-2 l \cos (\alpha) w \sin (\theta)}{r} \\
\frac{-5(\sqrt{3} \sin (\theta)+3 \cos (\theta)) l \cos (\alpha) w}{26 r} \\
\frac{-5(\sqrt{3} \sin (\theta)+3 \cos (\theta)) l \cos (\alpha) w}{26 r}
\end{array}\right] \\
\mathbf{t}_{r i}=\left[T_{r 1}, T_{r 2}, T_{r 3}\right]^{T}=\left[\begin{array}{c}
\frac{-2 w}{3} \\
\frac{w}{3} \\
\frac{w}{3}
\end{array}\right]
\end{gathered}
$$

In Equations (15) and (16), $w$ indicates the self-weight of the robot and is around $6.57 \mathrm{~N}$ without taking into account the cables. Two static phases are possible during the locomotion cycle of a robot. The first case is when one set of legs is clamped with pipeline walls and the central actuator remains in a fully retracted phase. The second case is when one set of legs is clamped with pipeline walls and the central actuator reaches its full extension. The value of $l$, which is essential to estimate the moments induced at the clamped ends, is essential for analysis and it can be identified by the CG positions of the robot for the two cases of central actuator position in CATIA software. The values of $l$ for the two cases were identified for 54 and $74 \mathrm{~mm}$ diameter pipelines, and are provided in Table 2.

Table 2. CG position (from CATIA) from the clamped legs of robot for fully retracted and extended positions of central actuator inside 54 and $74 \mathrm{~mm}$ diameter pipelines [15].

\begin{tabular}{ccc}
\hline $\begin{array}{c}\text { Position of } \\
\text { Central Actuator }\end{array}$ & $\begin{array}{c}\text { Radius of } \\
\text { the Pipeline (mm) }\end{array}$ & $\begin{array}{c}\text { Position of CG from } \\
\text { Clamped End (mm) }\end{array}$ \\
\hline Fully retracted & 27 & 128 \\
Fully extended & 27 & 149 \\
Fully retracted & 37 & 123 \\
Fully extended & 37 & 144 \\
\hline
\end{tabular}

For the estimation of forces, we considered that the pipeline is either horizontal ( $\alpha=0$ or $\pi$ radians) or vertical ( $\alpha=\pi / 2$ or $3 \pi / 2$ radians). The longitudinal and radial forces were estimated 
using Equations (15) and (16) for the two orientations of pipeline and the four CG positions in Table 2. Followed by this, using Equations (11)-(13), we estimated the normal forces as well as the actuator forces $F_{a}$. The results of the actuator forces for the two orientations of pipeline and four CG positions in Table 2 are represented in Figure 8.
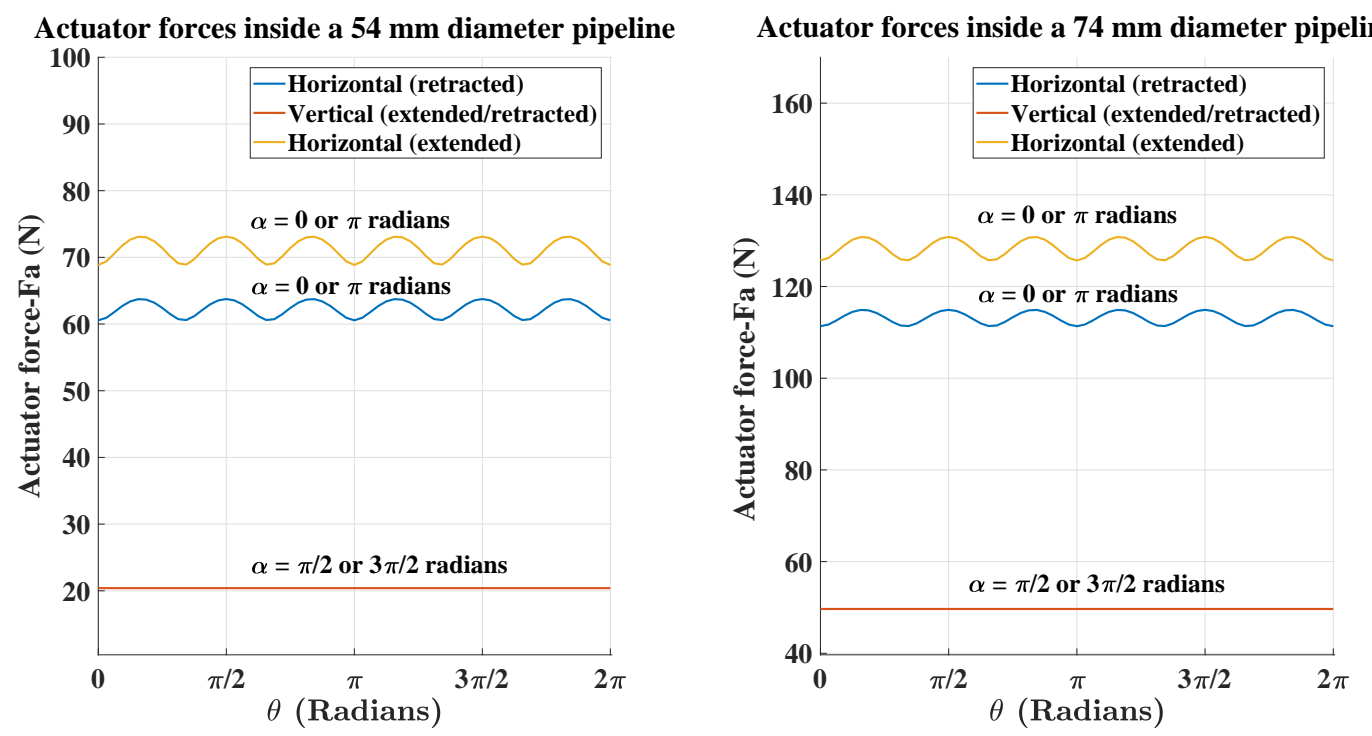

Figure 8. Front/Rear module actuator forces $F_{a}$ inside $54 \mathrm{~mm}$ (left) and $74 \mathrm{~mm}$ (right) diameter pipelines for horizontal ( $\alpha=0$ or $\pi$ radians) and vertical ( $\alpha=\pi / 2$ or $3 \pi / 2$ radians) orientations.

As shown in Figure 8, the forces were affected directly by the position of CG and the orientation of pipeline. For horizontal orientation of pipeline, varying range of actuator forces could be observed for both diameters. This Was caused mainly by the longitudinal force component, which is a function of the orientation of robot $(\theta)$. When the pipeline was vertical, no moment existed, as the longitudinal forces depended on the cosinus of the pipeline orientation $\alpha$ and these forces became zero at $\pi / 2$ and $3 \pi / 2$ radians. On the contrary, the radial forces continued to exist and they always remained a constant, as they depended only on the self-weight $w$ of the robot. Thus, at vertical orientation of pipeline, the actuator forces remained a constant due to the radial forces. For a given diameter, the highest amount of force was observed with the fully extended position of the central actuator with $\alpha$ at 0 or $\pi$ radians. As the actuator force $F_{a}$ depended on the factor $\eta_{f}$, which is a function of the pipeline radius, higher forces were observed for the $74 \mathrm{~mm}$ diameter pipeline. The force range values in Figure 8 are provided in Table 3.

Table 3. Front/Rear module actuator forces in Figure 8 inside horizontal and vertical orientations of pipeline for the CG positions in Table 2.

\begin{tabular}{cccc}
\hline $\begin{array}{c}\text { Position of } \\
\text { Central Actuator }\end{array}$ & $\begin{array}{c}\text { Pipeline } \\
\text { Radius (mm) }\end{array}$ & $\begin{array}{c}\text { Actuator Force (N) } \\
\text { Horizontal Pipe }\end{array}$ & $\begin{array}{c}\text { Actuator Force (N) } \\
\text { Vertical Pipe }\end{array}$ \\
\hline Fully retracted & 27 & $60.6-63.7$ & 20.4 \\
Fully extended & 27 & $68.8-73.1$ & 20.4 \\
Fully retracted & 37 & $111.3-115$ & 49.7 \\
Fully extended & 37 & $125.7-130.8$ & 49.7 \\
\hline
\end{tabular}

\section{Experimental Validation of the Prototype}

As shown in the previous sections, with the static models, it was possible to determine the actuator forces of front and rear modules by considering Model A or Model B (Figure 3). In addition, it allowed us to evaluate the performance of the motors necessary to achieve the robot's mobility. 
However, it is necessary to propose an algorithm that can adapt to variations of pipeline diameters. The force control concept makes it possible to detect variations in the motor's torque when it moves at constant speed. The peak results of a given pipeline diameter of static force model are employed in the control algorithm of the robot to attain static phases such as clamping or de-clamping during the locomotion. The motion profile of the locomotion cycle is similar to a "bang-bang" concept [24], which is optimal with respect to the execution times. In parallel, it is possible to reconstruct the current position of the robot by knowing the travel time and speed. The origins of the motor axes are taken by bending the legs to the maximum. The simpler form of the forward motion in pseudo-code is provided in Algorithm 1.

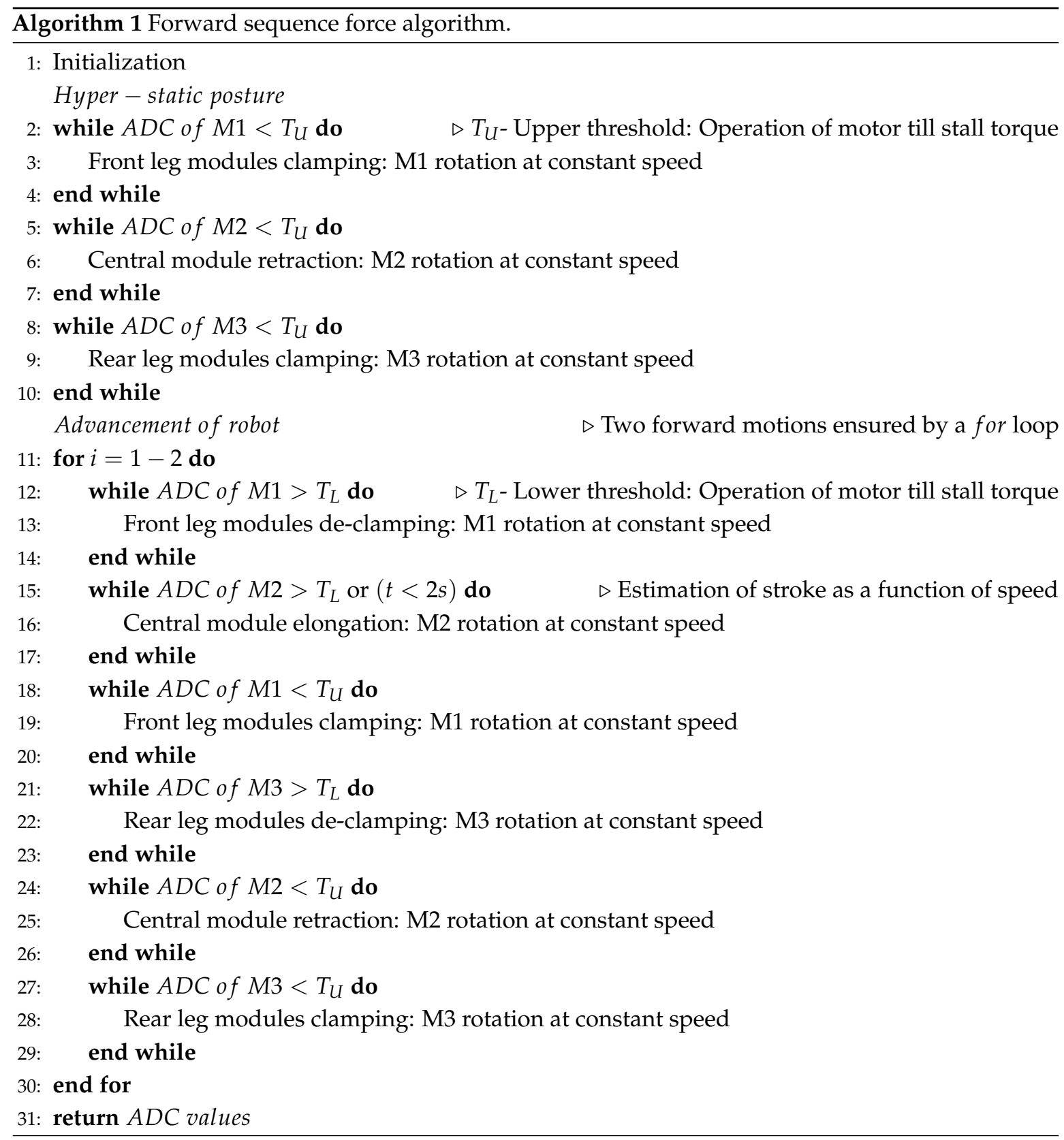

The upper and lower threshold limits $\left(T_{U}\right.$ and $\left.T_{L}\right)$ in the algorithm are voltage limits that are calibrated in accordance with the peak results of the numerical model to attain a static posture. At the end of the experiments, the robot was in an over-constrained posture. The front and rear leg 
de-clamping operations could be performed if necessary to withdraw the robot from the pipeline. A working model of the prototype made at LS2N laboratory is depicted in Figure 9.

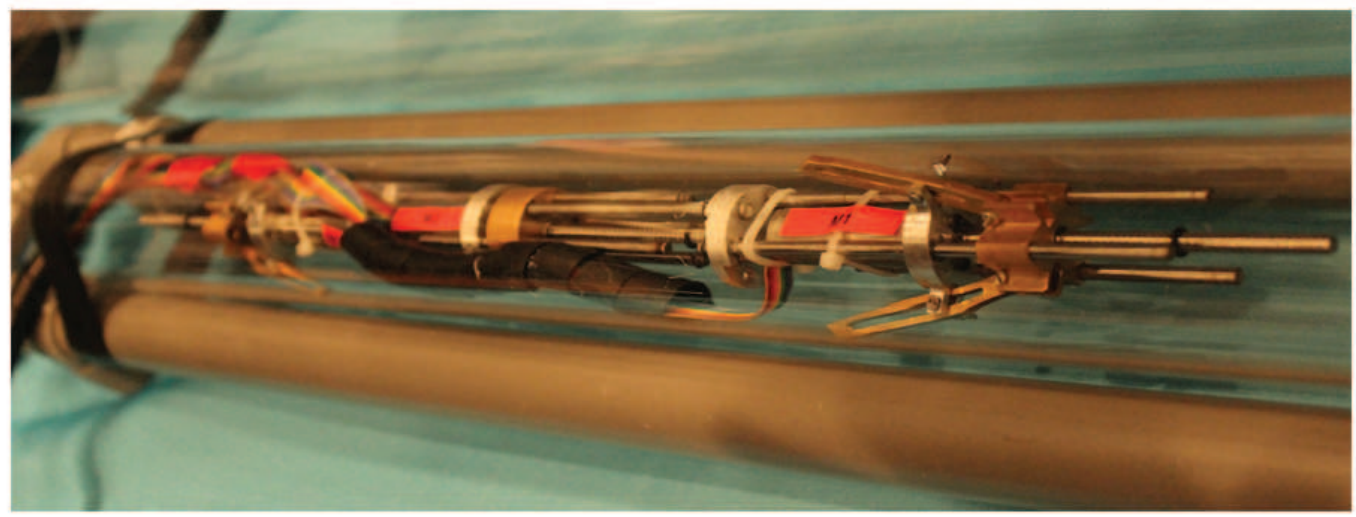

(A) Working of the robot inside the PVC test pipeline

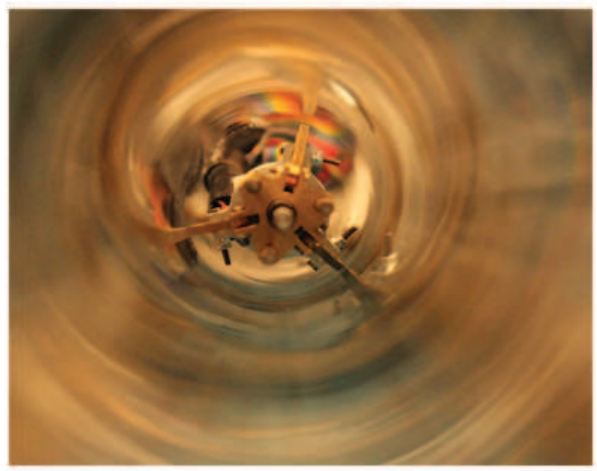

(B) Side view of the robot inside the pipe



(C) ESCON controllers and BeagleBone black

Figure 9. Prototype of the robot developed at LS2N, France (Photography copyrights: Vincent Jacques, Centrale Nantes, France).

In Figure 9, $A$ depicts the working model of the robot inside the test pipeline and $B$ represents the side view of the robot inside the pipeline. $C$ indicates the ESCON sensor board unit that is used for actuation of motors in the robot. The experimental setup consisted of a $74 \mathrm{~mm}$ diameter transparent PVC tube of $2.5 \mathrm{~m}$ length. With the help of $\mathrm{C}$ program, the following four sets of operations were conducted on the robot inside the pipeline with horizontal and vertical orientations:

- $\quad$ Forward motion (twice)

- $\quad$ Reverse motion (twice)

A BeagleBone (BB) black electronic board was employed to establish communication between the user interface of the computer to the motor control units. The BB black offers various pins such as the Pulse Width Modulation (PWM), General-Purpose Input/Output (GPIO), reconfigurable digital and Analog to Digital Converters (ADC) [25]. Two pins of the ESCON controller of each motor were connected to the BB black (PWM and ADC) for the control of motor speed and torques. Two other pins, one to the power source of $12 \mathrm{~V}$ and the other to the DC-motors were also used in the ESCON board. To control the voltage and current flow through the ESCON motors, the PWM was used to operate the motors under the required duty cycle with a control over the direction of rotation. The GPIO of BB operates on binary mode, which passes an ENABLE(1)/DISABLE(0) signal to the PWM. The PWM and GPIO of the BB have a maximum voltage of $3.3 \mathrm{~V}$. The PWM offers varying duty cycles, which were used to control the motor speed by controlling the voltage applied to the 
motors thereby preventing continuous motor running and losses. During idle stage, 50\% duty cycle was used as reference, wherein the current flow was 0 A. For safer running of motors, $80 \%$ duty cycle of the PWM was used during the clamping/retracting phases and 20\% duty cycle of the PWM was applied during the de-clamping/elongation phases at a period of $500 \mathrm{~ms}$. Thus, when a duty cycle was applied to a motor, the PWM generated an output voltage to the controllers to drive the motors. The speed constant, the nominal current and the maximum motor speed of the DC-motor specified in Table A1 were thus calibrated for the three motors individually [22] through their controllers in a computer before the start of experiments to rotate the motors at the desired velocity. For extracting the outputs, BB black's ADC was used. However, the maximum voltage supported by the ADC is $1.8 \mathrm{~V}$. For measuring the output torque of motors, the voltage range of ADC was calibrated in accordance with the nominal current of the motor. The detailed operating condition of BB black with the ESCON motor is provided in Table 4. There are also encoder inputs (EQEP) offered by BB black but they were not currently used in the control loop.

Table 4. BB black and ESCON controller settings for experiments.

\begin{tabular}{cccc}
\hline PWM Duty Cycle & ADC Voltage Range (V) & Nominal cUrrent Settings (A) & Motor Speed (rpm) \\
\hline $20 \%$ & 0 & -0.46 & 10,800 (Counter-clockwise) \\
$50 \%$ (idle) & 0.9 & 0 & 0 \\
$80 \%$ & 1.8 & 0.46 & 10,800 (Clockwise) \\
\hline
\end{tabular}

\subsection{Estimation of Currents and Forces of the Motors from Experiments}

To attain static phases, the threshold limits $T_{U}$ and $T_{L}$ were set as $1.5 \mathrm{~V}$ for the clamping/central retraction phases and $0.42 \mathrm{~V}$ for the de-clamping/central elongation phases. These values were set higher than the results of static force models because the weight of umbilicus and electromechanical factors were not considered in the numerical analysis. As the central actuator encounters lesser static forces as they have no masses attached to them, the same threshold limits were retained to attain larger displacements. The threshold values are also essential while operating under real-time conditions, where the exact diameter of pipelines are sometimes unknown. Lower values would have been more acceptable but the consumption of voltage by the motor at its start disrupts the calculation of generated force. This is due to the velocity profile used. In addition, the time of conversion by the $\mathrm{ADC}$ to the BB black is either quicker or lags behind. Thus, at the start of experiments, higher starting torque/current was usually observed. In such scenarios, the threshold values played an essential role for effective functioning of the motors and to cease them at the right moment. When threshold values were reached, the torque reached its stall point, which stopped the motor and the PWM was set to idle phase. The digital voltage values generated by the ADC of the BB were extracted and the results were plotted with the help of MATLAB. With the voltage values obtained during each step of the experiment, the current induced on the actuators was estimated by the equation:

$$
I=\frac{V_{t} I_{c}}{V_{i d l e}}-I_{\mathcal{c}}
$$

where $V_{t}$ indicates the voltage at a particular instant of time and $I_{\mathcal{C}}$ is the nominal current of motor in Table A1. $V_{\text {idle }}$ is the ADC voltage at 50\% PWM duty cycle, which was taken as $0.9 \mathrm{~V}$ according to Table 4 . The output power of the DC-motor was, however, affected by the power loss that was generated due to the current and resistance of the windings. The actual current generated from the motor is thus given by the equation:

$$
I_{a}=I-I_{n}
$$


where $I_{n}$ is the no-load current and this value is subtracted from Equation (17) to compensate the power loss. With the help of Equation (18), the output torques and corresponding forces can be calculated. The actual current generated from motors after power loss consideration is shown in Figure 10.

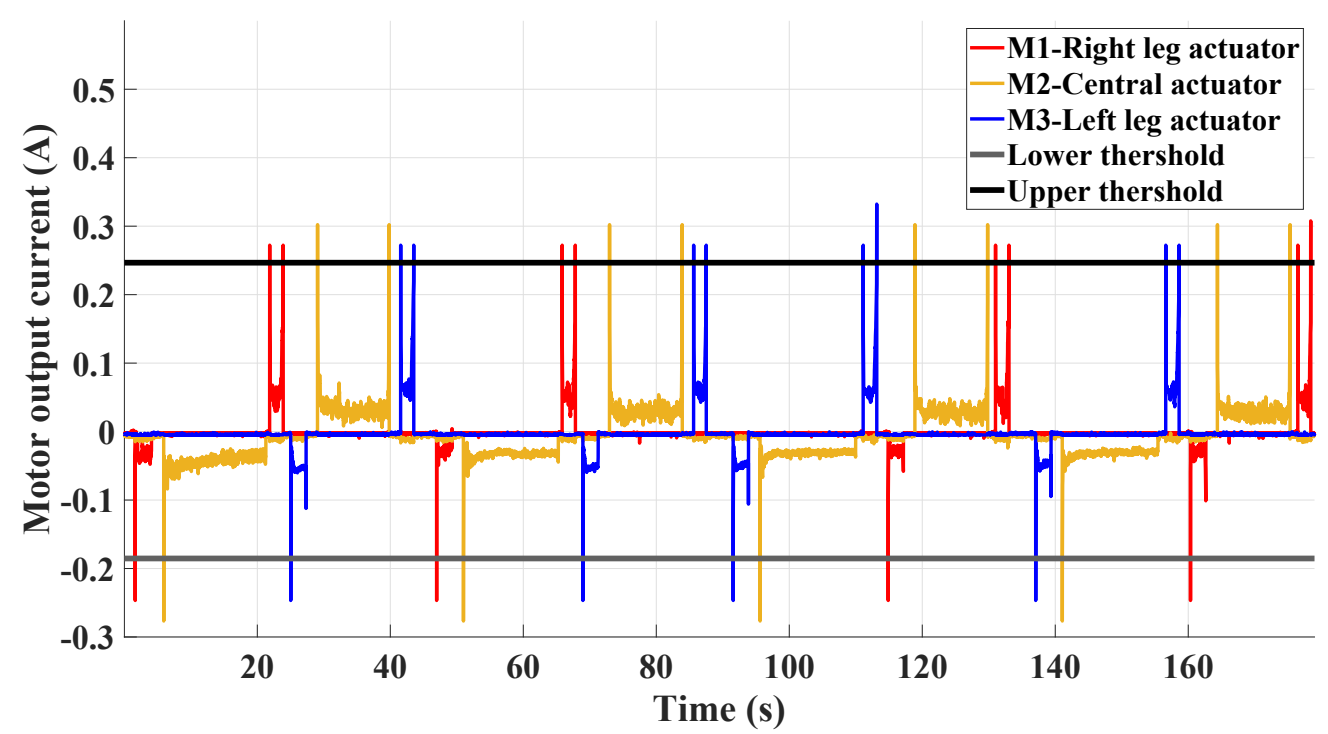

Figure 10. Output current of EC-motors from experiments.

In Figure 10, the red lines indicate the front leg module, the orange line indicates the central elongation module and the blue lines indicates the rear leg module. The threshold voltages are indicated in black and gray lines. The total time for performing two forward and reverse operations by the robot was around $179 \mathrm{~s}$. At $89.5 \mathrm{~s}$, two forward operations were completed and at $179 \mathrm{~s}$, the robot returned to the starting point. Higher noises were observed from the output voltages generated by the ADC. This was caused by some bending phenomena observed on the free end of the robot when the other end was clamped and also due to numerical errors. The frequency of the results was measured using MATLAB. With the help of Savitzky-Golay [26] filtering technique in MATLAB, the frequency from the ADC output was matched with the desired frequency to reduce the noise generated in the voltage. The output torques from the motors were then estimated by the equation:

$$
\tau_{M i}=I_{a} K_{T} G \eta_{m} \eta_{s} \quad \text { with } \quad i=1,2,3
$$

The torque constant $\left(K_{T}\right)$, reduction ratio $(G)$ and the efficiencies $\left(\eta_{m}\right.$ and $\left.\eta_{s}\right)$ were taken according to Tables A1 and A2, respectively. The operating force on the spindle drive of the motor can be estimated with the help of torque values obtained from Equation (19):

$$
F_{M i}=\frac{2 \pi \tau_{M i}}{p} \quad \text { with } \quad i=1,2,3
$$

In Equation (20), $p$ represents the screw pitch of the spindle drive, which is presented in Table A2. The output forces in the spindle drive were calculated using Equation (20) by performing the force control algorithm for horizontal and vertical orientations of pipeline. The results are represented in Figures 11 and 12. 


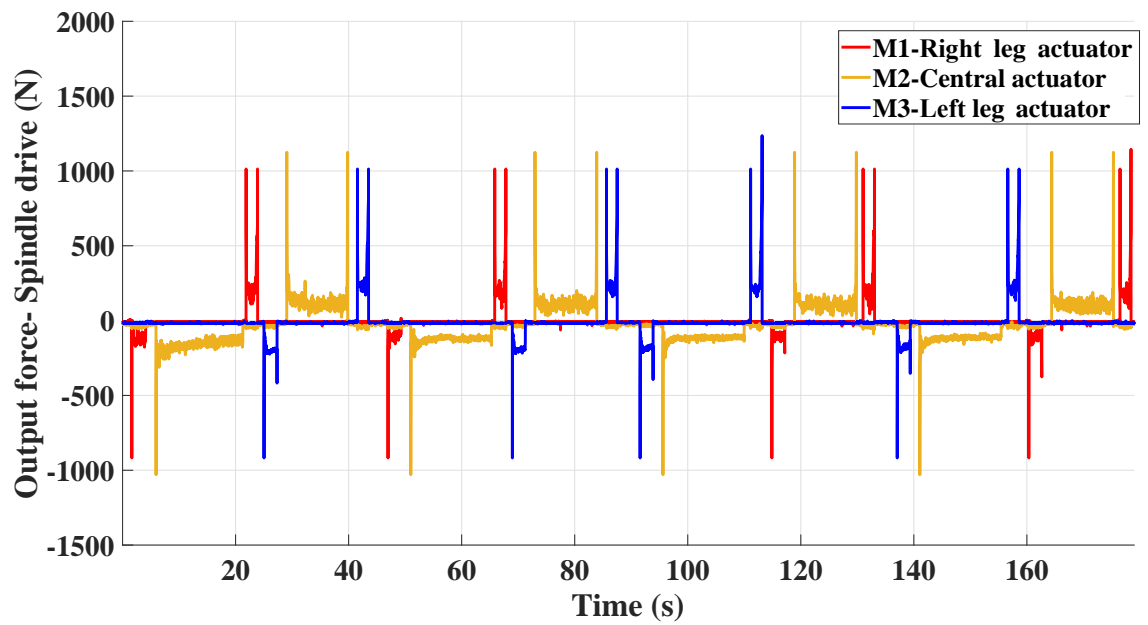

Figure 11. Experimental output forces in the spindle drive for horizontal orientation ( $\alpha=0$ or $\pi$ radians) of pipeline [27].

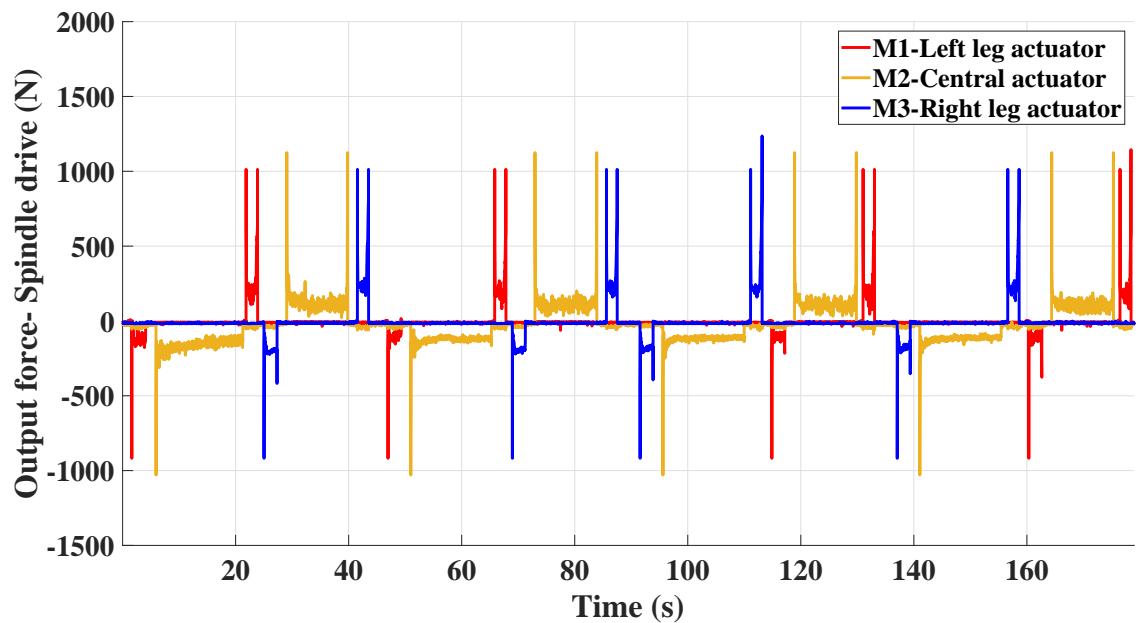

Figure 12. Experimental output forces in the spindle drive for vertical orientation $(\alpha=\pi / 2$ or $3 \pi / 2$ radians) of pipeline.

Since the velocity of the robot was too low $(0.43 \mathrm{~mm} / \mathrm{s}$ [17]), the lower threshold limit was set to a value lower than the peak limits of static force model such that the de-claming phase happened in a shorter duration. This can be observed in Figures 10-12. As the static forces on central module was lower, it extended/retracted to its maximum, which facilitated the robot covering larger distances during the locomotion cycle. The range of forces in Figures 11 and 12 for the three individual actuators are provided in Table 5.

Table 5. Measured value of experimental output forces on the spindle drive shown in Figures 11 and 12.

\begin{tabular}{ccccc}
\hline $\begin{array}{c}\text { Orientation of } \\
\text { Pipeline }\end{array}$ & Phase & $\begin{array}{c}\text { Initial } \\
\text { Force (N) }\end{array}$ & $\begin{array}{c}\text { Final } \\
\text { Force (N) }\end{array}$ & $\begin{array}{c}\text { Force under } \\
\text { Operation (N) }\end{array}$ \\
\hline Horizontal & Clamping (M1 \& M3) & 1000 & 1000 & $170-250$ \\
Horizontal & Declamping (M1 \& M3) & 900 & 0 & $80-150$ \\
Vertical & Clamping (M1 \& M3) & 1000 & 1000 & $160-330$ \\
Vertical & Declamping (M1 \& M3) & 900 & 0 & $100-180$ \\
Horizontal \& Vertical & Elongation (M2) & 1000 & 0 & $110-160$ \\
Horizontal \& Vertical & Retraction (M2) & 1100 & 1100 & $100-150$ \\
\hline
\end{tabular}


Higher forces of 1000-1100 N were observed at the start of clamping, elongation and retraction modules for both orientations of the pipelines. This was due to the fact that a constant velocity profile was used in the algorithm, which contributed to higher starting torque when a PWM duty cycle was applied. The inertial effects also contributed to these peak values. At the end of clamping operation in Motors M1 and M3, higher forces could be observed (Figures 11 and 12) over the de-clamping phases. This was caused by the leg masses and their flanges as they imposed more loads on the motor when they tried to establish contact with the walls of the pipeline. For the vertical orientation of pipeline, the forces under operation of clamping/de-clamping phases were found to be on the higher side over the horizontal orientation, especially in Motor M3. This was because Motor M3 was bolted along with M2 and, during vertical pass, the influence of gravity imposed more load on the motors, especially during the clamping phase. The umbilicus also contributed some forces significantly during vertical orientation of pipeline. It is interesting to note from the results in Figures 11 and 12 and Table 5 that the central actuator forces almost remained the same for both orientations of the pipeline. However, the forces under operation of central actuator was slightly higher for the horizontal orientation during elongation phase because the robot behaved similar to the cantilever beam assumption of static force model.

At the end of clamping phase, the contact force $F_{p}$ at the legs could be calculated with the help of Equation (13). Considering a $74 \mathrm{~mm}$ diameter tube for the experiments, a clamping force of around $630 \mathrm{~N}$ was required to establish a tight contact between the legs of the robot and the PVC test pipe. These forces tended to exist on the motor until the de-clamping phase began, where these forces became zero. The results are depicted in Figure 13.
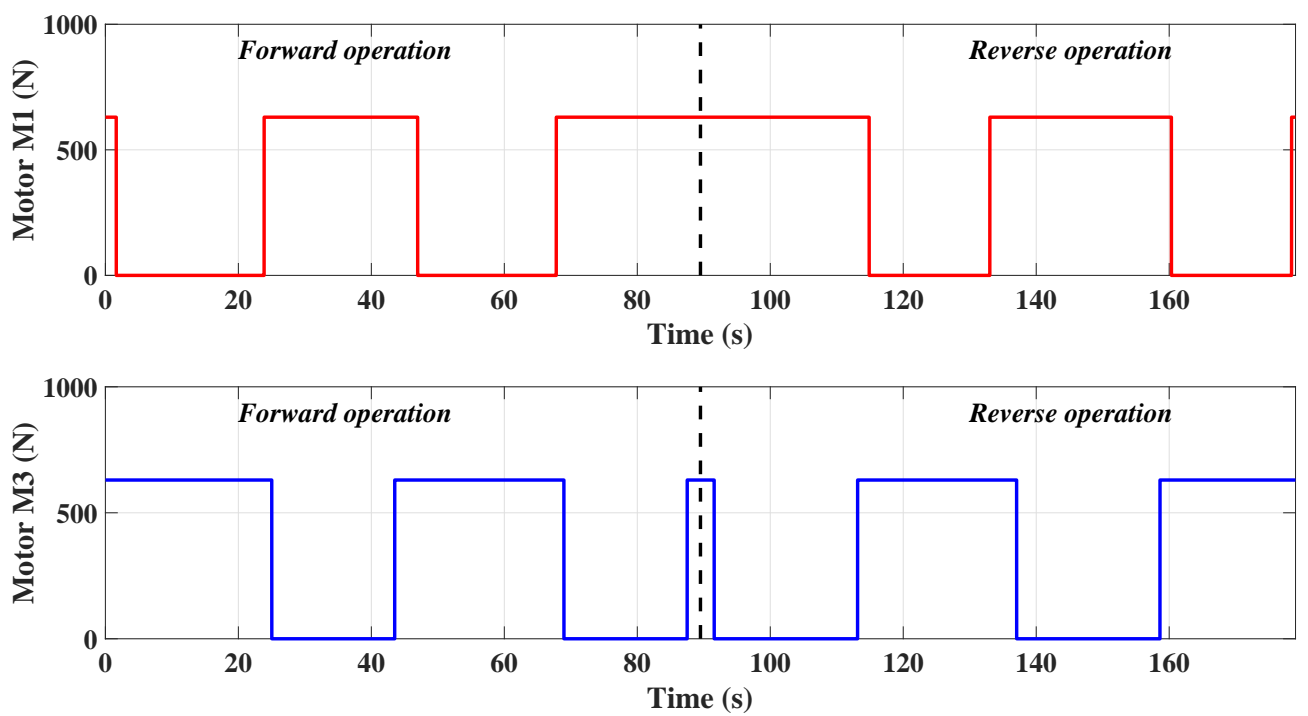

Figure 13. Clamping forces $\left(F_{p}\right)$ between legs of robot and PVC test pipe from experiments.

In Figure 13, we can observe that the clamping forces were higher when compared to the numerical model. Several factors contributed to this deviation. The numerical model considers a frictional contact between steel pipeline and bronze legs but the prototype was tested inside a PVC tube. In addition, with the numerical model, we could not take into account additional factors such as the umbilicus weight and electromechanical parameters. These reasons could have potentially contributed to higher force values in the experiments over the numerical model. 


\section{Discussions}

In this article, the static force model is presented for a bio-inspired caterpillar type inspection robot. Owing to the low velocity of the robot, a static model was sufficient to understand the clamping as well the static actuator forces of the robot. It was also possible to identify the key properties of motors that are required for the robot's locomotion cycle. The peak results coming from the numerical model allowed us to control the static phases on the prototype during experiments. In the numerical analysis, it was not possible to validate the electromechanical factors or frictional coefficients. Using a prototype, these factors were examined and we were able to isolate some differences from numerical analysis such as voltage fluctuations caused by the working condition of EC motors. The robot was able to overcome the issue of working inside pipelines less than $100 \mathrm{~mm}$ diameters as well as with varying diameters. The experiments were conducted for a $74 \mathrm{~mm}$ diameter pipe, however, it is also possible to carry out within a $54 \mathrm{~mm}$ diameter and then be compared with the static model. The results of experiments proved to be on the higher side owing to factors that were not considered in numerical analysis such as umbilicus weight and electromechanical factors. One major disadvantage of the robot is that it is a rigid model and it cannot be employed for other pipe profiles represented in Figure 6b-e. The spindle drive unit used in each module of the robot is currently oversized as it has a screw length of $102 \mathrm{~mm}$, which might be a problem when testing inside a pipeline with bends or curvatures.

In the future work, the initial problem that will be addressed is the modification of this robot into a reconfigurable mechanism. By retaining the caterpillar principle of the robot, a tensegrity type mechanism that uses three tension springs and a universal joint has been introduced as articulation units between each motor modules [28]. A detailed design and static analysis of this mechanism is presented in [29] to determine the geometrical workspace and bending angle limits, which are the key constraints taken into account for the robot to pass through bends or junctions. In the current design, standard parts of Maxon were used. By following an optimization procedure, the best dimensions for the motor will be determined that can allow it to pass through or overcome a curvature inside pipeline. The numerical model must be revisited for the reconfigurable robot with the tensegrity mechanism followed by which experiments must be conducted on the new prototype. An effective machine learning methodology is essential when it comes to the implementation of this robot for real time applications. A suitable lighting source has to be mounted on the robot when testing inside a steel pipeline to understand its position and also to determine the presence of cracks or openings. High velocity actuators will be proposed for the robot while testing inside longer pipelines in order to cover larger distances in a short interval of time. Replacement of existing BeagleBone black with the latest BeagleBone blue will be considered as the latter employs a lesser amount of cables and this could potentially address the cable management issue of the robot. In addition, some bio-inspired control techniques, as proposed by Arena et al. [30], will be considered for the control of the reconfigurable version.

Author Contributions: Development of static force model, Static force analysis, S.V. and D.C.; Digital mockup of Robot in CATIA, Development of prototype, D.C.; Experimental validation of prototype, S.V.; and Dynamic force analysis, F.B.

Funding: This project was partially funded by AREVA for the development of prototype.

Acknowledgments: We would like to thank Renaud Henry, Daniel Kanaan and Mathieu Porez for their participation in the study of this robot. In addition, we would like to thank Stéphane Jolivet of LS2N who helped in making the prototype of the robot and also we would like to thank Benjamin Ioller for his support on software programming.

Conflicts of Interest: The authors declare no conflict of interest. 


\section{Abbreviations}

The following abbreviations are used in this manuscript:

$\begin{array}{ll}\text { DKM } & \text { Direct Kinematic Model } \\ \text { IKM } & \text { Inverse Kinematic Model } \\ \text { CG } & \text { Center of Gravity } \\ \text { PWM } & \text { Pulse width modulation } \\ \text { GPIO } & \text { General-purpose Input/Output } \\ \text { ADC } & \text { Analog to Digital convertor } \\ \text { BB } & \text { BeagleBone }\end{array}$

Nomenclature

Symbol Description

$\rho \quad$ Leg module actuation units

$\rho_{c} \quad$ Central module actuation unit

$l_{1} \quad$ Length of leg used in slot-follower mechanism

$\theta \quad$ Rotation angle of robot

$\alpha \quad$ Rotation angle of pipeline

$\mathbf{R}_{z} \quad$ Rotation matrix about z-axis

$\mathbf{n}_{i} \quad$ Vector coordinates for $P_{i}$

$l \quad$ CG distance between clamped points and free end

$w \quad$ Self-weight of the robot

$\mathbf{f}_{i} \quad$ Force vector

$\mathbf{m}_{i} \quad$ Moment vector

${ }^{i} \tau_{0} \quad$ Wrench at CG

$T_{l i} \quad$ Tangential longitudinal force

$T_{r i} \quad$ Tangential radial force

$N_{i} \quad$ Normal forces

$\varphi \quad$ Coefficient of friction

$F_{a} \quad$ Magnitude of actuator force

$F_{p}, N_{\text {total }} \quad$ Magnitude of contact force/Total normal force

$\eta_{f} \quad$ Force transmission factor

I Current induced on actuators

$V_{t} \quad$ Magnitude of measured voltage at time $t$

$\tau_{\mathrm{Mi}} \quad$ Magnitude of torque measured in experiments

$p \quad$ Pitch of screw in spindle drive

$F_{M i} \quad$ Output force of spindle drive

\section{Appendix A}

Table A1. Specifications of the Maxon EC-motor (Part No. 283828) [22].

\begin{tabular}{cc}
\hline Parameters & Value \\
\hline Nominal voltage & $12 \mathrm{~V}$ \\
Nominal current $\left(I_{\mathcal{C}}\right)$ & $0.456 \mathrm{~A}$ \\
No load speed & $13,500 \mathrm{rpm}$ \\
No load current $\left(I_{n}\right)$ & $60.2 \mathrm{~mA}$ \\
Nominal speed & $5840 \mathrm{rpm}$ \\
Efficiency $\left(\eta_{m}\right)$ & $54 \%$ \\
Torque constant $\left(K_{T}\right)$ & $7.82 \mathrm{mNm} / \mathrm{A}$ \\
Speed constant $\left(N_{v}\right)$ & $1220 \mathrm{rpm} / \mathrm{V}$ \\
Terminal resistance & $15.7 \Omega$ \\
\hline
\end{tabular}


Table A2. Specifications of the Maxon Spindle drive (Part No. 424749) [22].

\begin{tabular}{cc}
\hline Parameters & Value \\
\hline Spindle screw diameter and pitch $(p)$ & $\phi 5 \times 2 \mathrm{~mm}$ \\
Reduction ratio $(G)$ & $455: 1$ \\
Max. static axial load & $500 \mathrm{~N}$ \\
Max. spindle length & $102 \mathrm{~mm}$ \\
Efficiency $\left(\eta_{s}\right)$ & $63 \%$ \\
\hline
\end{tabular}

\section{References}

1. Kassim, I.; Phee, L.; Ng, W.S.; Gong, F.; Dario, P.; Mosse, C.A. Locomotion techniques for robotic colonoscopy. IEEE Eng. Med. Biol. Mag. 2006, 25, 49-56. [CrossRef] [PubMed]

2. Takada, M. Self-Propelled Colonoscope. U.S. Patent 6,695,771, 24 February 2004.

3. Masuda, S. Apparatus for Feeding a Flexible Tube through a Conduit. UK Patent 1,534,441, 6 December 1978.

4. Hyun, J.; Hvung, J.; Young, M.; Juang, J.; Byungkyu, K.; Soo, H. Magnetic impact actuator for robotic endoscope. In Proceedings of the 32nd International Symposium on Robotics, Seoul, Korea, 19-21 April 2001; pp. 1834-1838.

5. Iddan, G.J.; Sturlesi, D. In Vivo Video Camera System. U.S. Patent 5,604,531, 18 February 1997.

6. Drapier, M.; Steenbrugghe, V.; Successeurs, B. Perfectionnements Aux Cathéters Médicaux. France Patent 1,278,965, 15 December 1961.

7. Ikuta, K.; Tsukamoto, M.; Hirose, S. Shape memory alloy servo actuator system with electric resistance feedback and application for active endoscope. In Proceedings of the 1988 IEEE International Conference on Robotics and Automation, Philadelphia, PA, USA, 24-29 April 1988; pp. 427-430.

8. Utsugi, M. Tubular Medical Instrument Having a Flexible Sheath Driven by a Plurality of Cuffs. U.S. Patent 4,148,307, 10 April 1979.

9. Treat, M.R.; Trimmer, W.S. Self-Propelled Endoscope Using Pressure Driven Linear Actuators. U.S. Patent 5,595,565, 21 January 1997.

10. Ginsburgh, I.; Carlson, J.A., III; Taylor, G.L.; Saghatchi, H. Method and Apparatus for Fluid Propelled Borescopes. U.S. Patent 4,735,501, 5 April 1988.

11. Nayak, A.; Pradhan, S. Design of a new in-pipe inspection robot. Procedia Eng. 2014, 97, $2081-2091$. [CrossRef]

12. Zhang, Y.; Zhang, M.; Sun, H.; Jia, Q. Design and motion analysis of a flexible squirm pipe robot. In Proceedings of the 2010 International Conference on Intelligent System Design and Engineering Application, Changsha, China, 13-14 October 2010; pp. 527-531.

13. Kwon, Y.S.; Lim, H.; Jung, E.J.; Yi, B.J. Design and motion planning of a two-moduled indoor pipeline inspection robot. In Proceedings of the 2008 IEEE International Conference on Robotics and Automation, Pasadena, CA, USA, 19-23 May 2008; pp. 3998-4004.

14. Henry, R.; Chablat, D.; Porez, M.; Boyer, F.; Kanaan, D. Multi-objective design optimization of the leg mechanism for a piping inspection robot. In Proceedings of the ASME 2014 International Design Engineering Technical Conferences and Computers and Information in Engineering Conference, Buffalo, NY, USA, 17-20 August 2014.

15. Chablat, D.; Venkateswaran, S.; Boyer, F. Mechanical Design Optimization of a Piping Inspection Robot. Procedia CIRP 2018, 70, 307-312. [CrossRef]

16. Rao, R.V.; Waghmare, G. A new optimization algorithm for solving complex constrained design optimization problems. Eng. Optim. 2017, 49, 60-83. [CrossRef]

17. Chablat, D.; Venkateswaran, S.; Boyer, F. Dynamic Model of a Bio-Inspired Robot for Piping Inspection. In ROMANSY 22-Robot Design, Dynamics and Control; Springer: Berlin/Heidelberg, Germany, 2019; pp. $42-51$.

18. Anthierens, C.; Ciftci, A.; Betemps, M. Design of an electro pneumatic micro robot for in-pipe inspection. In Proceedings of the IEEE International Symposium on Industrial Electronics (Cat. No.99TH8465), Bled, Slovenia, 12-16 July 1999; pp. 968-972. 
19. Anthierens, C.; Libersa, C.; Touaibia, M.; Bétemps, M.; Arsicault, M.; Chaillet, N. Micro robots dedicated to small diameter canalization exploration. In Proceedings of the 2000 IEEE/RSJ International Conference on Intelligent Robots and Systems (IROS 2000) (Cat. No.00CH37113), Takamatsu, Japan, 31 October-5 November 2000; pp. 480-485.

20. Venkateswaran, S.; Chablat, D. CATIA Model of the Robot and Its Simulation. Available online: https: / / www.youtube.com/watch?v=7z6-by83mtw (accessed on 6 March 2019).

21. Park, J.; Hyun, D.; Cho, W.H.; Kim, T.H.; Yang, H.S. Normal-force control for an in-pipe robot according to the inclination of pipelines. IEEE Trans. Ind. Electron. 2011, 58, 5304-5310. [CrossRef]

22. Maxon Motors, Program 2017/18. High Precision Drives and Systems. Available online: http://epaper. maxonmotor.com/ (accessed on 15 December 2017).

23. Ur-Rehman, R.; Caro, S.; Chablat, D.; Wenger, P. Multiobjective design optimization of 3-PRR planar parallel manipulators. In Proceedings of the 20th CIRP Design Conference, Nantes, France, 19-21 April 2010.

24. Khalil, W.; Dombre, E. Modeling, Identification and Control of Robots; Hermes Penton Ltd.: London, UK, 2002.

25. BeagleBone Black. Available online: https://elinux.org/Beagleboard:BeagleBoneBlack (accessed on 29 June 2018).

26. Savitzky-Golay Filtering. Available online: https://fr.mathworks.com/help/signal/ref/sgolayfilt.html (accessed on 28 August 2018).

27. Venkateswaran, S.; Chablat, D. Video of the Experiment on the Bio-Inspired Robot at 5x Speed. Available online: https:/ / www.youtube.com/watch?v=EPpZa-hvFKI\&t=4s (accessed on 10 April 2019).

28. Venkateswaran, S.; Chablat, D. A new inspection robot for pipelines with bends and junctions. In Proceedings of the 15th IFToMM World Congress, Krakow, Poland, 30 June-4 July 2019.

29. Venkateswaran, S.; Furet, M.; Chablat, D.; Wenger, P. Design and analysis of a tensegrity mechanism for a bio-inspired robot. In Proceedings of the ASME 2019 International Design Engineering Technical Conferences and Computers and Information in Engineering Conference, Anaheim, CA, USA, 18-21 August 2019.

30. Arena, P.; Fortuna, L.; Frasca, M. Attitude control in walking hexapod robots: An analogic spatio-temporal approach. Int. J. Circuit Theory Appl. 2002, 30, 349-362. [CrossRef]

(C) 2019 by the authors. Licensee MDPI, Basel, Switzerland. This article is an open access article distributed under the terms and conditions of the Creative Commons Attribution (CC BY) license (http://creativecommons.org/licenses/by/4.0/). 